\title{
NUEVA METODOLOGÍA PARA UN RANKING DE UNIVERSIDADES CHILENAS
}

\section{Introducción}

La educación superior es un caso típico de un sistema que funciona con información asimétrica. Postulantes y administradores del sistema tienen escasa información acerca del desempeño de las distintas universidades. Entonces, resulta de gran utilidad contar con indicadores acerca del desempeño relativo de sus instituciones. Como veremos, los actuales rankings de universidades adolecen de diversos problemas. Para subsanarlos, sugeriremos una metodología de construcción que refleje el desempeño relativo de las universidades y contribuya así a disminuir la asimetría inherente al sistema.

Esto es aún más importante en Chile, donde ha habido recientemente una notoria expansión del número de universidades -de 8 (1980) a más de 60-, de estudiantes universitarios -de 130.000 (1990) a casi 500.000 - y de profesionales titulados -de 13.000 (1990) a más de 35.000 egresados por año. Obviamente, esta rápida expansión ha dado origen a una demanda creciente por información acerca de estas instituciones.

Los jóvenes que ingresan a una universidad enfrentan un serio problema de información asimétrica. Los indicadores existentes para comparar universidades adolecen de una serie de deficiencias, como se verá más adelante. Prevalecen juicios sesgados, en los cuales hay, ciertamente, conflicto de intereses. La propaganda contribuye a la generación de información parcial y a veces distorsionada; ¿cuál es la relación (o el mecanismo de conexión) entre las fotos en las páginas sociales y la calidad de una universidad? Pero, incluso si los jóvenes 
decidieran adquirir la información pertinente para determinar en qué universidad les convendría estudiar, se encontrarían con el problema de que no pueden cotejar ni tampoco discriminar la calidad pedagógica de docentes de distintas universidades (no tienen elementos de juicio, especialmente al ingresar a la universidad).

Un joven que está por adoptar una decisión fundamental para su futuro necesita disponer de la mejor información posible para optimizar su toma de decisiones. La existencia de rankings de universidades hace más eficiente y menos costoso su proceso de búsqueda. Por lo pronto: (i) influencian la percepción que los jóvenes tienen sobre las distintas universidades y pueden afectar su decisión de elección, (ii) proporcionan información al aparato directivo de las universidades respecto del lugar que ocupa la universidad y sirven como mecanismo de planificación estratégica, (iii) permiten racionalizar el flujo de créditos para los estudiantes, y (iv) influyen sobre las empresas que contratan a los egresados universitarios (Webster, 2001).

Adicionalmente, indicadores que permitan comparar los resultados de la educación universitaria serían de gran utilidad para los administradores del sistema: les permitirían asegurar niveles básicos de calidad y entregar información acerca del funcionamiento de estas instituciones.

La publicación de rankings de universidades va a inducir cambios en el comportamiento de los participantes en el sistema. En consecuencia, estas tablas de posiciones no son permanentes: expresan lo observado en un momento específico y son indicativas de lo que sucedería si ninguna de las partes reaccionara ante la evidencia proporcionada.

En la primera sección se incluye una revisión crítica de los rankings tradicionales de universidades. En la segunda se utiliza el modelo multinivel para la elaboración de un nuevo método de ranking de universidades, modelo que incorpora el fenómeno del efecto grupal (peer effect). La tercera proporciona las estimaciones empíricas, 
utilizando dos cohortes de profesionales chilenos titulados en 1995 y 1998, y compara los rankings (entre carreras) para las dos cohortes; también se efectúa un análisis de la robustez de los resultados. La cuarta sección se refiere a aspectos generales del ranking; la quinta da cuenta de los rankings por carrera y la sexta aporta una síntesis de las principales conclusiones.

\section{Revisión crítica de los rankings tradicionales de universidades}

Actualmente, en Chile se elaboran rankings de universidades a partir de una combinación de distintos indicadores. Ejemplos de rankings de universidades chilenas son los elaborados por las revistas "Qué Pasa" (2004) y "El Sábado" (2004).

"Qué Pasa" utiliza los siguientes indicadores: (i) alumnado: incluye porcentaje de alumnos con Aporte Fiscal Indirecto (AFI), porcentaje de puntajes de excelencia, puntajes sobre 600 y 700 en la Prueba de Selección Universitaria (PSU); (ii) docencia: se mide a través de porcentaje de profesores jornada completa, alumnos por profesor y docentes con posgrado; (iii) infraestructura: considera número de alumnos por computador, inversión en infraestructura, recursos bibliográficos y metros por alumno; (iv) investigación: cuantificada por número de proyectos del Fondo de Desarrollo Científico y Tecnológico (Fondecyt), formación de magíster y número de publicaciones (estos indicadores se reportan individualmente).

Los indicadores utilizados por "El Sábado" son: (i) nivel de investigación: medido por el número de proyectos en ejecución y de publicaciones; (ii) composición de la matrícula: considerando el porcentaje de doctorados y magíster; (iii) calidad de los docentes: cuantificada a través del grado académico de éstos, la cantidad con dedicación a jornada completa y el número de alumnos por docente; (iv) calidad de los alumnos: medida por el número de alumnos con aporte fiscal indirecto y el porcentaje de éstos que obtuvieron más de 700 puntos en la PSU, y (v) infraestructura: según metros 
60 NUEVA METOdología PARA Un RANKING DE UNIVERSIDADES CHILENAS - Patricio Meller, David Rappoport

cuadrados disponibles para cada alumno, igualmente ponderados en la elaboración de los rankings.

Este constituye el marco metodológico tradicional de elaboración de un ranking de universidades. Hay varias críticas y de distinta índole a esta metodología:

(a) Ranking de universidades. Dada la división existente entre las facultades de una universidad, al escoger una carrera universitaria un joven no ingresa realmente a la universidad sino que a una facultad ${ }^{1}$. En consecuencia, una vez que ha definido sus preferencias respecto de la carrera que quiere estudiar, lo que necesita realmente es información específica referente a ésta.

Todas las carreras de una universidad no tienen por qué tener la misma calidad académica. Una universidad puede tener carreras de calidad académica alta, media y baja. Si se elaborara un indicador agregado de la calidad de esta universidad ¿qué realmente estaría midiendo? ¿Para que serviría? En otras palabras, ¿cuán relevante sería la información que le proporcionaría a un joven que quiere estudiar una carrera específica?

Supongamos que se elabora un ranking de universidades exclusivamente sobre la base del puntaje de los alumnos admitidos. Hay importantes diferencias en los puntajes de admisión de las diversas carreras. Las universidades chilenas tienen un distinto conjunto de carreras; además, hay gran varianza en el número de jóvenes admitidos en cada carrera por cada universidad. Luego, el resultado del ranking de universidades va a depender fundamentalmente de la composición de carreras de cada universidad y del número de alumnos admitidos en cada una de ellas. Esto ratifica la irrelevancia de este tipo de ranking.

Una investigación, orientada a examinar la varianza de ingresos de los profesionales universitarios, ha concluido que los diferenciales

1 Esto es aún más relevante en la educación superior chilena, debido a la presencia de diferentes sedes de las mismas universidades a través del país. 
de ingresos a través de las distintas carreras (universitarias) son significativamente mayores que los diferenciales de ingresos a través de las diversas universidades (Rappoport, 2004). En otras palabras, si un joven quiere escoger una profesión sobre la base del ingreso potencial (que tendría una vez que se titulara), debiera primero seleccionar la carrera y sólo después la universidad en que estudiaría dicha carrera.

En síntesis, los argumentos previos sugieren que los rankings de universidades deben hacerse separadamente para cada carrera universitaria.

(b) Ranking usando insumos (inputs). Los indicadores utilizados para la elaboración de los rankings citados son en su mayoría insumos o recursos utilizados en el proceso docente; por ejemplo, alumnos por profesor, docentes con posgrado, libros/computadores/metros cuadrados por alumno.

Sólo para efectos explicativos, supongamos que la educación universitaria es un proceso productivo en que a un joven se le aplica un conjunto de estos insumos y el output final es el profesional universitario. Lo que realmente interesa es la calidad de este joven profesional, es decir, ¿cuán bueno es un profesional que egresa de una universidad específica?

Por otro lado, el uso de los insumos de los que dispone cada universidad, ies un buen predictor de la calidad de sus egresados? Diversas opiniones parecen dar una respuesta negativa a esta interrogante: (i) al utilizar un insumo específico, es necesario explicar el mecanismo a través del cual este insumo afecta a la calidad del egresado. Por ejemplo, ¿cuál es el nivel óptimo de libros/ computadores/metros cuadrados por alumno que maximiza la calidad de un profesional egresado? (ii) Dado que se tienen distintos tipos de insumos, ¿cómo se combinan éstos para tener un indicador agregado? Por ejemplo, ¿cómo se suman el indicador alumnos por profesor con el indicador computadores por alumno? 
62 NUEVA METOdología PARA Un RANKING DE UNIVERSIDADES CHILENAS - Patricio Meller, David Rappoport

Entonces, es imposible establecer el conjunto "correcto" de insumos y de sus ponderaciones respectivas para obtener el indicador agregado. Además, no hay estudios que muestren cuál es la sensibilidad de los rankings resultantes ante variaciones de los insumos seleccionados y de las ponderaciones que les han sido asignadas.

(c) Cuestionamiento al uso de rankings. Considerando que el número de universidades chilenas es de alrededor de 60, ¿tiene realmente sentido elaborar un ranking que las ordene?

El presidente de la Universidad de Stanford se preguntaba respecto del ranking publicado por US News \& World Report: "ison realmente diferentes la primera universidad (del ranking) de la segunda, o incluso de la décima?" (Casper, 1996). En el caso chileno, ¿cuál es la diferencia en calidad de una universidad "rankeada" en el lugar 10 de una que ocupa el lugar 15 o 20? ¿Cuán útil o determinante es para un joven saber que las universidades que quiere escoger están "rankeadas" en los lugares $5^{\circ}$ y $6^{\circ}$ ? ¿Es estable el ranking temporalmente?

Aún más, los rankings son presentados como si el ordenamiento en cuestión fuera único y verdadero. Pero, ¿cuál es la validez estadística de uno de 60 universidades?

No obstante lo anterior, una encuesta efectuada en EE.UU. a 220.000 estudiantes respecto del "efecto que había tenido en su decisión de elección de un college en particular la existencia del ranking de universidades", proporcionó las siguientes respuestas: $11 \%$ encontró el ranking muy importante, 30\% lo consideró algo importante y el 59\% no le asignó ninguna importancia. Se debe considerar que los estudiantes que encontraron importante el ranking eran muy buenos alumnos y provenían de familias de altos ingresos, con padres con educación universitaria (McDonough, 1997, citado en Provan y Abercromby, 2000).

(d) Nuevo indicador alternativo al ranking tradicional. Este nuevo indicador tiene tres elementos distintivos: (i) uso de una variable única que está relacionada al output, lo que resuelve los problemas asociados 
al uso de insumos; además, se elimina el problema de agregación y de las ponderaciones relativas de diferentes insumos. (ii) El ranking es sustituido por categorías o clases de universidades. (iii) La clasificación de una universidad en una de estas categorías es determinada de manera no ambigua y puede ser verificada estadísticamente.

Parece lógico pensar que los indicadores que estarían más próximos a medir la calidad de las universidades serían los de desempeño u output. La universidad supuestamente ejerce una función fundamental en la determinación del nivel de ingresos de sus egresados. Un objetivo de este artículo es la elaboración de un ranking de universidades chilenas construido sobre la base del ingreso de sus titulados. Dado el uso de información individual de los egresados de las universidades, ha sido posible estimar los diferenciales del ingreso medio de éstos y verificar su validez estadística. Además, se ha controlado por tipo de carrera, género, experiencia y edad.

El hecho de usar como variable fundamental para la clasificación de las universidades el ingreso laboral de sus titulados va a inducir al equipo directivo de estas instituciones a considerar los factores que operan en el lado de la demanda de profesionales. Hasta hace poco las universidades latinoamericanas y sus académicos han definido el currículo de las carreras y su tasa de expansión desde el lado de la oferta, generalmente con total prescindencia del futuro mercado laboral que los profesionales egresados enfrentarán, esto es, lo que les sucederá en su vida profesional. Esta actitud puede haberse agravado en el caso chileno, en que se ha desarrollado un mercado muy competitivo; incluso las universidades estatales enfrentan requerimientos significativos de autofinanciamiento, por lo cual los ingresos provenientes de la matrícula y aranceles constituyen un mecanismo importante de generación de recursos.

Por último, cuando se utilizan indicadores de desempeño (output) con el fin de medir la calidad, es necesario controlar por las variables que determinan el desempeño para aislar el efecto de la universidad. Este punto ha sido ampliamente discutido en la 
literatura de indicadores de valor agregado educacional (Goldstein, 2001 y Wilson, 2003). En el caso de considerarse los ingresos de los titulados, sería conveniente incorporar en el análisis las características que los determinan, tales como experiencia, nivel socioeconómico, sexo, sector de actividad económica, entre otros.

Como se señaló, el ranking de universidades será sustituido por el uso de categorías de universidades. Se clasificarán las universidades en tres clases: primera $(\star \star \star)$, segunda $(\star \star)$ y tercera $(\star)$. Una universidad será clasificada como de primera clase $(\star \star \star)$ si tiene un ingreso promedio relativamente mayor al de todos los titulados de la misma carrera, considerando todas las universidades presentes en la muestra. De manera análoga, será clasificada como de tercera clase $(\star)$ si tiene un ingreso promedio relativamente menor al de todos los titulados de la misma carrera. Por último, será clasificada como de segunda clase $(\star \star)$ si tiene un ingreso promedio similar al del grupo de todos los titulados de esa carrera.

Para un conjunto de 60 universidades, desde el punto de vista informativo (en otras palabras, mayor contenido de información) es mucho más útil un sistema de categorías con tres clases de universidades que la existencia de un ranking completo de las mismas. En efecto, la información que indica que la universidad z, x, o w ocupa el décimo lugar del ranking (entre las 60) es menos informativa que la que señala que esta universidad $z, x, 0 \mathrm{w}$ es de segunda clase $(\star \star)$. Además, la clasificación a través del número de $\star$ es más fácil de retener.

La analogía de las universidades con los hoteles puede resultar ofensiva; sin embargo, permite ilustrar las ventajas de esta metodología de categorías en un contexto con un grave problema de información asimétrica. Cuando un viajero llega a una ciudad que desconoce y está buscando un hotel para pasar la noche, le es muy útil contar con una lista de hoteles con sus precios y el número de estrellas asociado a cada hotel. En este caso, el número de estrellas es un mejor indicador de calidad que el precio. Si escoge un hotel de 
cuatro estrellas puede imaginarse la calidad del hotel seleccionado; lo mismo le sucede si escoge un hotel de una o dos estrellas.

A nuestro juicio, los jóvenes que están decidiendo qué carrera universitaria estudiar y en qué universidad reducirían bastante su desconocimiento (y asimetría de información) respecto de las alternativas que enfrentan si cada carrera universitaria indicara el número de estrellas que tiene (o la clase a la que pertenece). Esto debiera estar incluido en todos los folletos, propaganda, avisaje (e incluso en las fotos de las páginas sociales) de las carreras universitarias. Obviamente, una misma universidad puede tener carreras con uno, dos o tres estrellas.

El sistema de acreditación de carreras universitarias constituye en realidad una aplicación simplificada y dicotómica de esta metodología. Las carreras universitarias acreditadas tendrían una estrella y las no acreditadas ninguna. Sin embargo, a nuestro juicio, dado que existe una gran varianza en la calidad (y en los ingresos de sus titulados), es conveniente una diferencia adicional entre las carreras universitarias acreditadas.

\section{Modelo multinivel ${ }^{2}$}

En general, la información relacionada con los titulados de las universidades contiene datos acerca de la universidad y de la carrera. Una forma natural de estructurar dicha información es considerar dos niveles: los titulados de una misma carrera -nivel 1- agrupados en distintas universidades -nivel 2. En este contexto llamamos unidades a los integrantes de cada nivel, siendo unidades de nivel 1 los titulados, y unidades de nivel 2 las universidades. Las unidades en un nivel se consideran agrupadas en unidades del siguiente nivel. De esta forma, consideramos a los titulados de una misma carrera agrupados por universidades. Para el presente estudio interesa la

2 Para una presentación formal más completa sobre el modelo multinivel ver Rappoport et al., 2004. 
66 NUEVA METOdología PARA Un RANKING DE UNIVERSIDADES CHILENAS - Patricio Meller, David Rappoport

forma en que esta estructura organizacional afecta los ingresos de los titulados, esperándose que entre los titulados de una misma carrera los egresados de una universidad particular tengan desempeños similares en el mercado laboral ${ }^{3}$.

La figura 1 presenta la estructura de la información. Bajo este esquema, las universidades que imparten dos carreras son consideradas como dos universidades distintas, pues cada carrera es considera separadamente.

Al considerar la información de los titulados de las universidades, un problema metodológico es el de sesgo de selección. Éste se produce cuando las muestras en los distintos grupos considerados no son aleatorias (Heckman \& Robb, 1985).

Figura 1: Estructuración de la información de titulados de una misma carrera.

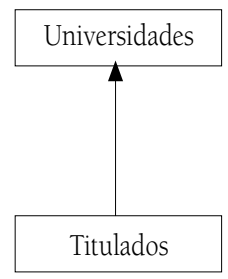

En otras palabras, la comparación de dos universidades está influenciada por el hecho de que los alumnos de cada una son diferentes respecto a características que influyen en los ingresos observados. Este problema es especialmente serio cuando se intenta cuantificar los efectos causales que la universidad genera en los ingresos de sus titulados. El problema de sesgo de selección se reduce en el caso del enfoque multinivel, porque estima las diferencias entre los ingresos (promedio) de los titulados entre las universidades; este modelo supone que los efectos diferenciales de las universidades

3 Este tipo de modelos puede ser extendido para realizar comparaciones, ya sea entre carreras, entre universidades o entre ambas simultáneamente; además, es posible analizar los factores determinantes de estas clasificaciones (Goldstein, 1995). 
sobre los ingresos de sus titulados siguen una distribución normal. O sea, las estimaciones del modelo multinivel no están orientadas a apreciar el efecto (causal) de la educación universitaria sobre los ingresos, sino que determinan en qué medida la varianza residual se debería a la universidad (Goldstein, 1995). Luego, en el caso de nuestro análisis y debido a la ausencia de otros controles, las estimaciones son respecto del titulado con las características promedio de los controles omitidos. En síntesis, en la medida en que el efecto "universidad" siga la distribución especificada, las estimaciones del modelo multinivel serían apropiadas.

Los distintos efectos generados por la educación universitaria apuntan en la dirección de posicionar a las universidades en diversas categorías de ingreso. En primer lugar, si consideramos esta educación como un proceso que permite aumentar los ingresos de sus titulados, entonces las diferencias relativas de calidad de enseñanza se debieran reflejar en las mismas diferencias en los ingresos de sus titulados. En segundo término, si percibimos la educación universitaria como un mecanismo de señalización de las habilidades relativas (Spence, 1974), entonces, las universidades que proporcionan señales "convincentes" de transmisión de mayores habilidades relativas se posicionarían relativamente mejor en la distribución de ingresos. En tercer lugar, la presencia de un efecto grupal (peer effect) podría reforzar la posición de las universidades a lo largo de la distribución de los ingresos. El efecto de los compañeros podría darse, ya sea a través del aumento de la productividad del aprendizaje debido a la educación universitaria, donde los compañeros y las interacciones de éstos y los docentes jugarían un rol preponderante; adicionalmente, la creación de redes de trabajo genera un catalizador de redes sociales que proporciona conexiones y puede mejorar la inserción en el mercado laboral. En ambos casos, las universidades que logren captar a los grupos de estudiantes relativamente mejores conseguirían posicionarse en mejor forma en los rankings. En síntesis, existen varios mecanismos alternativos que permitirían a las universidades posicionarse en las clasificaciones elaboradas según el ingreso de sus titulados. 
Para la confección de los rankings de universidades utilizamos la información de ingresos de los titulados individualmente, lo que nos permite usar una mayor cantidad de información y evaluar su validez estadística. Para esto se aplica un modelo que estima los diferenciales medios de ingresos entre universidades.

Suponemos una relación estadística lineal entre el (logaritmo del) ingreso y las variables que lo determinan,

$$
y_{i j}=x_{i j} \beta+u_{j}+\varepsilon_{i j}
$$

donde $y_{i j}$ es el (log del) ingreso del titulado i egresado de la universidad $j, x_{i j}$ es el vector fila con las características que determinan el ingreso, $\beta$ es un vector de parámetros asociados a esas características, $u_{j}$ es el término aleatorio asociado a la universidad $j$, y $\varepsilon_{i j}$ es el término aleatorio asociado al titulado $i$ de la universidad $j$. La especificación de la distribución del término aleatorio es un elemento distintivo entre los modelos de regresión y multinivel ${ }^{4}$. El modelo multinivel es una alternativa de estimación cuando se tienen observaciones interdependientes; éste es el caso cuando existe un efecto grupal.

\section{Aplicación empírica del modelo multinivel}

Esta sección presenta los resultados de las estimaciones del modelo multinivel para las carreras universitarias: Contador Auditor, Educación de Párvulos, Ingeniería Comercial, Periodismo y Psicología. La estimación de los diferenciales de ingresos medios entre universidades es relativamente más eficiente según el modelo multinivel que de acuerdo con el tradicional de regresión. El primero captura una mayor cantidad de diferencias estadísticamente significativas entre carreras universitarias, aumentando la información contenida en las categorías elaboradas.

4 Para una discusión más extensa respecto de la comparación entre el modelo multinivel y el modelo de regresión, ver Rappoport et al., 2004. 
La información básica corresponde a registros individuales de titulados de carreras universitarias en 1995 y 1998; la variable utilizada corresponde a los ingresos percibidos al segundo y al quinto año postitulación ${ }^{5}$. Estos registros representan casi el 90\% del universo de los titulados en esos años para las carreras consideradas.

La información para cada titulado incluye además sexo, edad, cohorte, carrera y características de la universidad. El ingreso ha sido obtenido del Servicio de Impuestos Internos, según las declaraciones de los individuos y preservando la confidencialidad de la información. La variable ingreso corresponde al ingreso real bruto mensual de los titulados en pesos de junio de $2004^{6}$. Por último, en la base se reportan la región, naturaleza jurídica y año de fundación de las universidades.

En Chile existen dos grandes grupos de universidades: las que pertenecen al Consejo de Rectores de las Universidades Chilenas (CRUCH) y las que no pertenecen a éste ${ }^{7}$. Entre las primeras se distinguen las universidades tradicionales y las universidades derivadas. Las tradicionales fueron fundadas con anterioridad a 1980. Posteriormente, luego de la reforma a la educación superior realizada en 1981, se crearon nuevas universidades, muchas de ellas constituidas a partir de las sedes regionales de las universidades tradicionales: universidades derivadas (todas las estatales creadas a partir de 1981 incluso las que no derivan de universidades tradicionales). Las universidades que no pertenecen al CRUCH son todas privadas y han sido fundadas a partir de 1982.

Se han agrupado las universidades que poseen características similares, de modo de mantener la confidencialidad de la información; además, esto aumenta el número de observaciones en los grupos

5 Los ingresos corresponden a los años 1997 y 2000, para la cohorte 1995, y 2000 y 2003 para la cohorte 1998.

6 Para mayor información sobre el cálculo de los ingresos y los filtros aplicados para la selección de las observaciones, ver Futuro Laboral (2004).

7 El CRUCH se compone mayoritariamente de universidades estatales, pero contiene algunas pocas universidades privadas. 
considerados. Serán usadas tres variables para agrupar las universidades en distintas categorías: naturaleza jurídica (tradicionales, derivadas y privadas), localización geográfica (regiones Metropolitana y $\mathrm{V}^{8}$, en adelante RM y V, y regiones I, IV y VI, XII, en adelante regiones) y antigüedad (fundadas antes o después de 1981). De esta forma es posible analizar la existencia de un "premio" por reputación para las primeras universidades privadas y/o para las tradicionales respecto de las derivadas.

Se obtienen siete grupos de universidades ${ }^{9}$ : (1) tradicionales de la RM y V; (2) tradicionales de regiones; (3) privadas antiguas de la RM y V; (4) privadas nuevas de la RM y V; (5) privadas nuevas de regiones; (6) derivadas de la RM y V, y (7) derivadas de regiones. Como se señaló, esta clasificación permite incrementar el número de observaciones al interior de los grupos analizados ${ }^{10}$; al tener un número suficiente de observaciones es posible verificar la significancia estadística de la clasificación de las carreras universitarias en las distintas categorías.

\section{Elementos generales del ranking de carreras universitarias}

Una vez establecidos estos grupos de universidades, restringimos el análisis a las carreras universitarias donde existen titulados en todos los grupos de universidades ${ }^{11}$.

Con respecto a una carrera universitaria específica, los rankings se elaboran sobre la base de las estimaciones del diferencial del (log del) ingreso medio entre los distintos grupos de universidades;

8 El supuesto implícito es que hay un alto grado de sustitución entre las universidades de estas dos regiones.

9 Para una metodología de agrupación de universidades diferente, ver Brunner (2006).

10 Esta clasificación además permite mantener la confidencialidad de las universidades consideradas en la base

11 En la base utilizada se identifican 58 grupos de carreras universitarias bajo tres criterios: (i) ingresos; (ii) contenidos temáticos; y (iii) duración (ver www.futurolaboral.c]) 
esta variable (entre los titulados) es la variable dependiente para la aplicación del modelo multinivel.

La clasificación de las carreras universitarias en las tres diferentes categorías se realiza según la estimación del diferencial medio del logaritmo del ingreso para cada universidad y su desviación estándar (Goldstein y Spiegelhalter, 1996). Como muestra la figura 2, una universidad donde el diferencial estimado no es estadísticamente distinto de cero al 95\% de confianza -el intervalo de confianza incluye al cero- será clasificada como de segunda clase $(\star \star)$. Cuando la estimación del diferencial medio del logaritmo del ingreso es positiva (negativa) al 95\% de confianza la universidad será clasificada como de primera (tercera) clase.

Adicionalmente, se proporcionan los porcentajes medios de alumnos que tienen ingresos por sobre el promedio de la carrera. Este indicador enfatiza el importante papel que desempeñan las variables omitidas -como los talentos individuales- en la determinación del ingreso. Como muestra la evidencia, titularse de una universidad relativamente mejor (peor) que la universidad promedio no es equivalente a obtener un ingreso por sobre (bajo) el promedio de todos los titulados de la carrera.

Figura 2. Esquema para clasificación de carreras universitarias

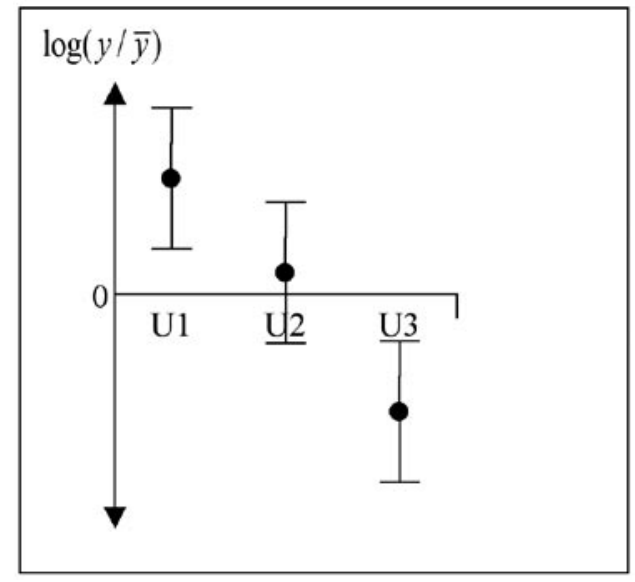


72 NUEVA METOdOlogía PARA Un RANKING DE UNIVERSIDADES CHILENAS - Patricio Meller, David Rappoport

\section{Rankings por carrera}

A continuación, elaboramos los cinco rankings de universidades chilenas correspondientes a las carreras de Contador Auditor, Educación de Párvulos, Ingeniería Comercial, Periodismo y Psicología. Como se verá, tanto la posición en el ranking como la magnitud de los diferenciales medios en los niveles de ingresos varían en las carreras consideradas.

Los rankings elaborados para cada carrera según el ingreso de los titulados utilizan el modelo multinivel (ecuación 1), considerando los ingresos al quinto año postitulación en ambas cohortes juntas (1995 y 1998). Posteriormente, se examinan las diferencias en los rankings obtenidos al considerar los ingresos al segundo año postitulación teniendo en cuenta ambas cohortes juntas (1995 y 1998). Además, se analizan las discrepancias en los rankings al contemplar separadamente cada cohorte de titulados.

Los rankings generados representan la percepción que tiene el mercado de las universidades consideradas. Pero las diferencias observadas pueden estar influenciadas por las características tanto de las universidades como de los titulados. Un ejemplo relativo a las primeras es el distinto énfasis puesto en la formación respecto de las habilidades demandadas por el mercado; entre los relativos a los rasgos de titulados destaca la habilidad natural de estos ${ }^{12}$. En consecuencia, los rankings podrían no reflejar exclusivamente la calidad relativa de las universidades; las habilidades específicas de los titulados afectan los resultados observados.

La tabla 1 muestra la clasificación que se establece mediante las estimaciones del modelo multinivel para las cinco carreras, al considerar los ingresos al quinto año postitulación. Al considerar los más de 1.900 titulados de contador auditor se obtiene el ranking de universidades reportado en la primera columna de la tabla 1. En

12 Las medidas de habilidad natural de los jóvenes podrían operacionalizarse con los puntajes en las pruebas de Selección a la Universidad (PSU o Prueba de Aptitud Académica, PAA). 
Contador Auditor los grupos de universidades que pertenecen a la primera clase $(\star \star \star)$ son tradicionales y privadas antiguas de la RM y V. Los titulados de estas universidades tienen un nivel de ingresos promedio $32 \%$ mayor respecto del ingreso de todos los titulados. Al mismo tiempo, un $74 \%$ tiene ingresos por sobre el promedio de la carrera $^{13}$. Sólo el grupo de universidades privadas nuevas de regiones pertenecen a la tercera clase $(\star)$, con un ingreso promedio un $42 \%$ más bajo que el de todos los titulados. En el caso de los titulados de este grupo de universidades tan sólo un $24 \%$ posee ingresos por sobre el promedio.

Tabla 1: Rankings de universidades por carrera según los ingresos al $5^{\circ}$ año postitulación (ambas cohortes)

\begin{tabular}{|c|c|c|c|c|c|}
\hline & Contador Auditor & Educación de Párvulos & Ingeniería Comercial & Periodismo & Psicología \\
\hline $\begin{array}{l}\text { Primera } \\
\text { clase }(\star \star \star)\end{array}$ & $\begin{array}{l}\text { U. Tradicional-RMyV } \\
\text { U. Privada-antigua- } \\
\text { RMyV }\end{array}$ & $\begin{array}{l}\text { U. Privada-antigua- } \\
\text { RMyV }\end{array}$ & $\begin{array}{l}\text { U. Tradicional-RMyV } \\
\text { U. Privada-antigua- } \\
\text { RMyV }\end{array}$ & $\begin{array}{l}\text { U. Tradicional-RMyV } \\
\text { U. Privada-antigua- } \\
\text { RMyV }\end{array}$ & $\begin{array}{l}\text { U. Tradicional-RMyV } \\
\text { U. Privada-antigua- } \\
\text { RMyV }\end{array}$ \\
\hline $\begin{array}{l}\text { Segunda } \\
\text { clase }(\star \star)\end{array}$ & $\begin{array}{l}\text { U. Derivada-RMyV } \\
\text { U. Tradicional- } \\
\text { regiones } \\
\text { U. Derivada-regiones } \\
\text { U. Privada-nueva- } \\
\text { RMyV }\end{array}$ & $\begin{array}{l}\text { U. Tradicional-RMyV } \\
\text { U. Derivada-RMyV U. } \\
\text { Tradicional-regiones } \\
\text { U. Derivada-regiones } \\
\text { U. Privada-nueva- } \\
\text { RMyV }\end{array}$ & $\begin{array}{l}\text { U. Derivada-RMyV } \\
\text { U. Tradicional- } \\
\text { regiones } \\
\text { U. Privada-nueva- } \\
\text { RMyV }\end{array}$ & $\begin{array}{l}\text { U. Derivada-RMyV } \\
\text { U. Derivada-regiones } \\
\text { U. Privada-nueva- } \\
\text { RMyV } \\
\text { U. Privada-nueva- } \\
\text { regiones }\end{array}$ & $\begin{array}{l}\text { U. Derivada-RMyV } \\
\text { U. Derivada-regiones } \\
\text { U. Privada-nueva- } \\
\text { RMyV }\end{array}$ \\
\hline $\begin{array}{l}\text { Tercera } \\
\text { clase }\left(^{\star}\right)\end{array}$ & $\begin{array}{l}\text { U. Privada-nueva- } \\
\text { regiones }\end{array}$ & $\begin{array}{l}\text { U. Privada-nueva- } \\
\text { regiones }\end{array}$ & $\begin{array}{l}\text { U. Derivada-regiones } \\
\text { U. Privada-nueva- } \\
\text { regiones }\end{array}$ & $\begin{array}{l}\text { U. Tradicional- } \\
\text { regiones }\end{array}$ & $\begin{array}{l}\text { U. Tradicional- } \\
\text { regiones }\end{array}$ \\
\hline
\end{tabular}

Fuente: Elaboración propia en base a Futuro Laboral (2004).

La segunda columna de la tabla 1 muestra el ranking confeccionado con los casi 1.000 titulados de Educación de Párvulos. En este caso, sólo el grupo de universidades privadas antiguas de la RM y $\mathrm{V}$ pertenecen a la primera clase ( $\star \star \star)$, con un ingreso un $16 \%$ por sobre el promedio y un $65 \%$ de sus titulados con ingresos sobre la media. A su vez, sólo el grupo de universidades privadas nuevas

13 Para la información detallada y los cálculos específicos de este caso y los siguientes, ver Rappoport et al. (2004). 
de regiones pertenecen a la tercera clase $(\star)$, con un ingreso de $15 \%$ bajo el promedio y sólo un 35\% de sus titulados con ingresos sobre la media.

En la misma tabla, la tercera columna reporta el ranking elaborado para Ingeniería Comercial, según los más de 2.700 titulados considerados. Los grupos de universidades de la RM y V, tradicional y privadas antiguas, pertenecen a la primera clase $(\star \star \star)$, con un diferencial medio de ingresos de $21 \%$ y una fracción de sus titulados con ingresos por sobre el promedio de $65 \%$. En la tercera clase ( $\star$ ) se ubican las de regiones derivadas y privadas nuevas, con un diferencial medio de ingresos de $25 \%$ y una fracción de titulados bajo la media de ingresos de $31 \%$.

El ranking para los más de 750 titulados de Periodismo se informa en la cuarta columna de la tabla 1 . En la primera clase ( $\star \star \star)$ se ubican las universidades de la RM y V tradicional y privadas antiguas, con ingresos un $19 \%$ por sobre el promedio y un $61 \%$ de titulados sobre la media. En la tercera clase $(\star)$ se ubican las tradicionales de regiones, con un ingreso promedio $16 \%$ más bajo que el promedio y un $42 \%$ de titulados bajo el promedio de la carrera.

Finalmente, el ranking para los cerca de 900 titulados de Psicología es reportado en la quinta columna de la tabla 1. Pertenecen a la primera clase $(\star \star \star)$ los grupos de universidades de la RM y V tradicional y privadas antiguas. Estos titulados poseen un ingreso un $12 \%$ por sobre el promedio, y un $58 \%$ de ellos posee ingresos sobre la media. A la tercera clase $(\star)$ pertenecen las tradicionales de regiones, con un diferencial de ingreso promedio de $-14 \%$ y una fracción de titulados por sobre la media de $28 \%$.

Las estimaciones de los rankings elaborados podrían ser cuestionadas en cuanto a su validez, debido a la elección del modelo multinivel como la herramienta más apropiada de estimación. Para validar el uso de este modelo se ha aplicado el test de razón de verosimilitudes, que permite rechazar, al 1\% de confianza, la hipótesis 
nula de varianza cero interuniversidades $\left(\sigma_{u}^{2}=0\right)$. Esto implica una ventaja relativa del modelo multinivel sobre el modelo de un solo nivel o modelo de regresión. Además, como muestran Rappoport et al. (2004), los rankings estimados son robustos al considerar separadamente cada cohorte de titulados (1995 y 1998) o los ingresos al segundo año postitulación. En síntesis, la evidencia sugiere que sería apropiado utilizar un modelo multinivel para la elaboración de rankings de universidades en este contexto.

\section{Conclusiones}

En primer lugar, se observa que tanto el ranking como la magnitud de los diferenciales medios de ingresos calculados para los siete grupos de universidades dependen de la carrera considerada, reafirmando la necesidad de elaborar rankings separadamente por carrera y no por universidades. En efecto, en Contador Auditor se observan los mayores diferenciales de ingreso entre las universidades de la primera $(\star \star \star)$ y tercera ( $\star$ ) categoría respecto del promedio de la carrera: $32 \%$ y $-42 \%$, respectivamente. Mientras que en Psicología los respectivos diferenciales estimados son de $12 \%$ y $-14 \%$.

Lo anterior muestra que la universidad desempeña una importante función en la determinación de los ingresos de sus titulados.

Se observa que existe un premio por reputación para las universidades ubicadas en la RM y V. Efectivamente, al considerar las universidades privadas de estas regiones, en las cinco carreras consideradas, las privadas antiguas de la RM y V se ubican siempre por sobre las privadas nuevas de estas regiones. Además, en cuatro de las cinco carreras consideradas, las universidades tradicionales de la RM y V se ubican por sobre las derivadas de estas regiones. En el caso de las universidades de regiones no se observa un predominio de las tradicionales; ello no necesariamente invalida el premio por reputación mencionado ya que las derivadas de regiones corresponden en muchos casos a las antiguas sedes de las tradicionales, lo que les 
76 NUEVA METOdOlogía PARA Un RANKING DE UNIVERSIDADES CHILENAS - Patricio Meller, David Rappoport

permite gozar una larga historia, aun cuando fueron fundadas después de las tradicionales.

Por último, cabe enfatizar que los rankings elaborados representan la percepción del mercado laboral. Dadas las restricciones de información, no es posible separar realmente las calidades (docentes) relativas de los grupos de universidades analizados de las características naturales específicas de los respectivos titulados.

\section{Referencias bibliográficas}

Brunner, José Joaquín (2006) Sistema privatizado y mercados universitarios: competencia reputacional y sus efectos. Documento de trabajo, Escuela de Gobierno, Universidad Adolfo Ibáñez.

Casper, Gerhard (1996) Carta privada a J. Fallows, 23 de septiembre. [Sitio en Internet] Disponible en http://www.stanford.edu/dept/pres-provost president/speeches/961206gcfallow.htm

Diario El Mercurio, Revilsta El Sábado (2004) No 322, noviembre.

Goldstein, Harvey (1995) Multilevel Statistical Models, $3^{\text {rd }}$ edition New York: Oxford University Press.

Goldstein, Harvey y Spiegelhalter, David (1996) League tables and their limitations: Statistical issues in comparisons of institutional performance. Journal of the Royal Statistical Society. Series A (Statistics in Society), 159 (3), pp. 385-409.

Heckman, James y Robb, Richard (1985) Alternative methods for evaluating the impact of interventions. En: Heckman, James y Singer, Burton (eds.) Longitudinal Analysis of Labour Market Data. New York: Wiley.

McDonough, Patricia; Lising, Anthony; Walpole, Antonio et al. (1998) College rankings: democratized college knowledge for whom? Research in Higher Education, 39 (5), pp. 513-537.

Provan, David y Abercromby, Karen (2000) University league tables and rankings: a critical analysis, CHEMS paper $\mathrm{N}^{\circ} 30$, December.

Qué Pasa (2004), Ranking de universidades, Santiago de Chile, noviembre.

Rappoport, David (2004) Elección de una carrera universitaria y el ingreso monetario esperado. Tesis para optar al grado de magíster en economía 
aplicada. Facultad de Ciencias Físicas y Matemáticas, Universidad de Chile.

Rappoport, David; Benavente, José Miguel; Meller, Patricio (2004) Ranking de universidades chilenas según los ingresos de sus titulados. Documento de trabajo $\mathrm{N}^{\circ}$ 306, Banco Central de Chile.

Spence, Michel (1974) Market signaling: information transfer in hiring and related processes. Cambridge: Harvard University Press.

Webster, Thomas (2001) A principal component analysis of the U.S. News and World Report tier rankings of colleges and universities. Economics of Education Review, 20 (3), pp. 235-244.

Wilson, Deborah (2003) Which ranking? The use of alternative performance indicators in the English secondary market. CMPO Working Paper Series $N^{\circ} 03 / 058$, University of Bristol.

Recibido: 6 de octubre de 2006

Aceptado: 23 de octubre de 2006 